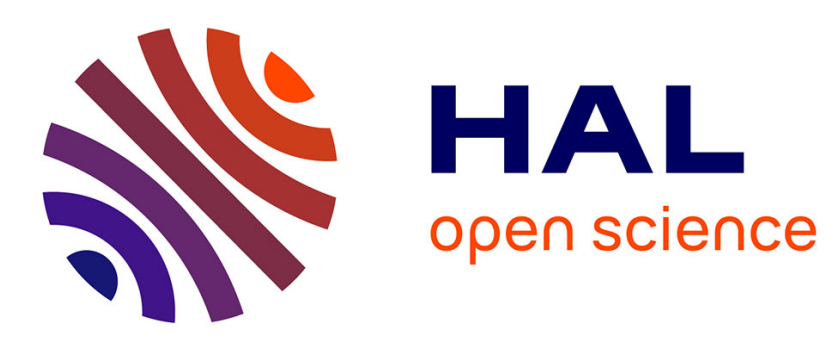

\title{
Kinematics of a bubble freely rising in a thin-gap cell with additional in-plane confinement
}

Lucas Pavlov, M. Verónica d'Angelo, Mario Cachile, Véronique Roig, Patricia Ern

\section{- To cite this version:}

Lucas Pavlov, M. Verónica d'Angelo, Mario Cachile, Véronique Roig, Patricia Ern. Kinematics of a bubble freely rising in a thin-gap cell with additional in-plane confinement. Physical Review Fluids, 2021, 6 (9), pp.093605. 10.1103/PhysRevFluids.6.093605 . hal-03414109

\section{HAL Id: hal-03414109 \\ https://hal.science/hal-03414109}

Submitted on 4 Nov 2021

HAL is a multi-disciplinary open access archive for the deposit and dissemination of scientific research documents, whether they are published or not. The documents may come from teaching and research institutions in France or abroad, or from public or private research centers.
L'archive ouverte pluridisciplinaire HAL, est destinée au dépôt et à la diffusion de documents scientifiques de niveau recherche, publiés ou non, émanant des établissements d'enseignement et de recherche français ou étrangers, des laboratoires publics ou privés. 


\section{Open Archive Toulouse Archive Ouverte}

OATAO is an open access repository that collects the work of Toulouse researchers and makes it freely available over the web where possible

This is an author's version published in: http://oatao.univ-toulouse.fr/28393

\section{Official URL:}

https://doi.org/10.1103/PhysRevFluids.6.093605

\section{To cite this version:}

Pavlov, Lucas and D'Angelo, M. Verónica and Cachile, Mario and Roig, Véronique and Ern, Patricia Kinematics of a bubble freely rising in a thin-gap cell with additional in-plane confinement. (2021) Physical Review Fluids, 6 (9). 093605. ISSN 2469-990X

Any correspondence concerning this service should be sent to the repository administrator: tech-oatao@listes-diff.inp-toulouse.fr 


\title{
Kinematics of a bubble freely rising in a thin-gap cell with additional in-plane confinement
}

\author{
Lucas Pavlov $\odot,{ }^{1,2}$ M. Verónica D’Angelo, ${ }^{1,2}$ Mario Cachile $\circledast,{ }^{1,2}$ \\ Véronique Roig ${ }^{3},{ }^{3}$ and Patricia Em $0^{3, *}$ \\ 'Universidad de Buenos Aires, Facultad de Ingenieria, Grupo de Medios Porosos, Buenos Aires, Argentina \\ ${ }^{2}$ CONICET; Buenos Aires, Argentina \\ ${ }^{3}$ Institut de Mécanique des Fluides de Toulouse, Université de Toulouse and CNRS, Toulouse. France
}

\begin{abstract}
We analyze the behavior of air bubbles freely rising at high Reynolds numbers in a planar thin-gap cell filled with distilled water. The gapthickness of the cell is fixed to $h \simeq 2.8 \mathrm{~mm}$ (or $h \simeq 1 \mathrm{~mm}$ in additional experiments) and its in-plane width $W$ is varied from 2.4 to $21 \mathrm{~cm}$. This allows us to investigate the evolution from unconfined thin-gap situations (i.e., large $W$ and $h \ll W$ ) controlled by the bubble characteristic lengths (diameter in the cell plane $d>h$ and thickness close to the gap size $h$ ) to doubly confined situations controlled by the channel dimensions. As the bubble size $d$ increases, and beyond a critical value that depends on $W$, we observe a mean rise velocity of the bubble, $V_{b}$, lower than that for larger $W$, along with a modification of the bubble shape. The departure occurs for oscillating bubbles of approximate elliptical shape, which becomes closer to circular as the lateral confinement increases. We further investigate how the bubble oscillatory motion is impacted by the transverse confinement. Assuming that the wall effect is related to the strength of the downward fiow generated by the bubble, we introduce the relative velocity $U_{\text {rel }}=V_{b} / \xi$, where $\xi=1-d / W$ is the confinement ratio and found $U_{\text {rel }} \simeq V_{b, \infty}$ for all the cell widths considered, where $V_{b, \infty}$ is the mean rise velocity in the absence of the transverse confinement (i.e., for $W$ sufficiently large). This provides an estimation, at leading order, of the bubble velocity, $V_{b} \simeq 0.8 \xi(h / d)^{1 / 6} \sqrt{g d}$, that generalizes the expression proposed by Filella $e t$ al. J. Fluid Mech. 778, 60 (2015) and accounts for the additional drag experienced by the bubble due to the lateral walls. We then show that, for given $d$ and $\xi$, the frequency and amplitudes of the oscillatory motion can be predicted using the characteristic length and velocity scales, $d$ and $U_{\text {rel }}$. As the bubble size is increased further, the bubble behavior becomes fully dominated by the channel dimensions. Cylindrical-capped shapes emerge, corresponding to a radius of curvature $R_{c}$ at the front of the bubble, $R_{c} \simeq 0.31 \mathrm{~W}$, independent of the bubble size and of the gap thickness. At the same time, the mean rise velocity of the bubble saturates at a constant value, corresponding to a constant Froude number, $\mathrm{Fr}=V_{b} / \sqrt{g W}$, that depends on the gap thickness $h$ of the cell.
\end{abstract}

\section{INTRODUCTION}

The complex motion of bubbles freely rising in a thin-gap cell at high Reynolds numbers has focused growing interest in the past decades, as it combines several fundamental and practical interests. In particular, mixing or mass transfer, which are looked for in applications involving confined bubbles reactors, preserves in the thin-gap configuration specific properties associated with inertial

*Corresponding author: Patricia.Ern@imft.fr 
flows, while operating limited volumes of liquid (e.g., Refs. [1-5]). The planar geometry of the cell also renders more accessible the measure of the bubble behavior and of the perturbation generated in the surrounding liquid. However, though specific applications involve planar geometries [6], in most situations, the flow induced by the rising bubble is in practice also bounded in the plane of the cell by lateral walls. The aim of the present paper is, therefore, to investigate the influence of an additional transverse confinement on the bubble behavior, by varying the experimental device progressively from a two-dimensional cell to a channel of a rectangular cross section.

The hydrodynamical interaction between a freely moving bubble and a wall is a problem that has received particular attention in the case of three-dimensional bubbles. In fact, the no-slip condition imposed by the wall on the flow field produced by the bubble results in a modification of the flow field about the bubble and, therefore, in a modification of the hydrodynamical loads experienced by the bubble during its rise. These in turn affect the shape and motion of the bubble. Depending on the relative distance of the bubble to the wall, and on the bubble size, repulsive or attractive behaviors from or to the wall can be observed [7-9]. In the case of a small bubble confined between parallel walls, the interaction can result in a periodic path of the bubble, which oscillates in-between the two walls [10]. Introducing additional walls is, therefore, a way to investigate the coupling between the motion and the shape of a freely rising single bubble, by modifying the strength and structure of the liquid velocity field about the moving bubble.

The inertial regime of bubbles freely rising in a thin-gap cell was investigated in detail by Roig et al. [11]. They highlighted in particular the existence of different types of bubble motion and provided a characterization of the different paths observed for increasing bubble sizes. Filella et al. [12] further pointed out the influence of the gap thickness $h$ on the mean vertical velocity of the bubble $V_{b}$ and the effect of the bubble's shape on the characteristics of its oscillatory motion. By comparing their results for a cell with $h \simeq 3 \mathrm{~mm}$ to those of Roig et al. [11] for $h \simeq 1 \mathrm{~mm}$ and the same couple of fluids (water and air), Filella et al. [12] proposed a simple generic estimation for the mean rise velocity of the bubble $V_{b}$ valid for a large range of bubble sizes, $V_{b, \infty} \simeq 0.7 \sqrt{g d_{\mathrm{eq}}}$, which can also be expressed as

$$
V_{b, \infty} \simeq k(h / d)^{1 / 6} \sqrt{g d}
$$

where $k=0.75$ and $V_{b}$ is denoted $V_{b, \infty}$ for consistency with the remainder of the paper. In this expression, $g$ is the gravitational acceleration, and the diameters

$$
d_{\mathrm{eq}}=\left(3 d^{2} h / 2\right)^{1 / 3} \text { and } d=\sqrt{4 \mathcal{A} / \pi}
$$

are, respectively, the three-dimensional equivalent diameter of the bubble calculated with its volume $\left(d_{\text {eq }}\right)$ and the planar equivalent diameter of the bubble determined from the area $\mathcal{A}$ covered by the bubble in the plane of the cell $(d)$. Expression (1) indicates that the mean vertical velocity of the bubble $V_{b}$ is not only proportional to the gravitational velocity $\sqrt{g d}$ but also depends on the parameter $h / d$ imposed on the bubble by the small gap. Hashida et al. [13] generalized the expression to the case of air bubbles rising through stagnant glycerol-water solutions in a 3-mm-gap cell, introducing a dependence of $V_{b}$ on the Morton number characterizing the couple of fluids. Hashida et al. [14] further extended the correlation to a slurry composed of fine silica particles. Piedra et al. [15] and Wang et al. [16] performed experiments in thin-gap cells and confirmed the influence of $h$ on $V_{b}$. However, the experimental results from Wang et al. [16,17] provide values for $V_{b}$ departing from those of Roig et al. [11], a variation that could be attributed to the differences in the other dimensions of the cells. The following questions can, therefore, be asked: does the cell width affect the mean rise velocity of the bubble $V_{b}$, when does the influence of lateral walls become significant, and what is the magnitude of this effect? Preliminary answers can be found in the theoretical prediction by Collins [18] in the potential flow limit for two-dimensional bubbles or in the viscous regime investigated by Murano and Okumura [19], both indicating a decrease in the bubble rise velocity. The purpose of the present paper is to evaluate experimentally this effect over 


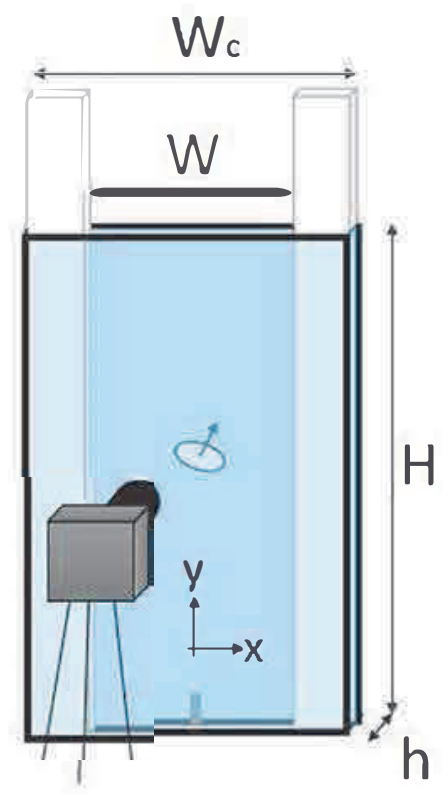

FIG. 1 . Scheme of the experimental setup, consisting in a thin-gap cell (thickness $\boldsymbol{h}$, height $H$, and width $W_{c}$ ), in which internal walls can be included in order to reduce the effective width to $W$.

a large range of control parameters corresponding to bubbles at high Reynolds numbers, including oscillating bubbles associated with periodic vortex shedding in their wake.

The paper is organized as follows. Section II presents the experimental devices and describes the experimental tools used to characterize the bubble kinematics and shape. Section III provides an overview of the different types of paths observed when the cell width $W$ changes. For a large range of bubble sizes, Sec. IV then presents the effect of the in-plane confinement on the mean rise velocity of the bubble and on its mean deformation. Sections V and VI then focus on the impact of the confinement on specific regimes of bubble behavior, corresponding to oscillating or cylindrical-capped bubbles, respectively. The paper ends with concluding remarks (Sec. VII).

\section{EXPERIMENTAL TOOLS}

We here present the experimental setup and the recording technique used to measure the bubble kinematics. The experimental apparatus consisted of a thin-gap cell of thickness $h$, height $H$, and width $W_{c}$ oriented vertically and filled with distilled water (see Fig. 1). Polymethyl methacrylate (PMMA) plates were included inside the cell as internal lateral walls to reduce the effective width from $W_{c}$ to $W$. Air bubbles were released from a syringe connected to a nozzle located in the center at the bottom of the cell. Experiments were performed at ambient temperature of $(19 \pm 1)^{\circ} \mathrm{C}$. Three different cells were used, all of them with $h \simeq 2.8 \mathrm{~mm}$. The first one was an acrylic (PMMA) cell with $h=(2.7 \pm 0.1) \mathrm{mm}, H=30 \mathrm{~cm}$, and $W_{c}=9 \mathrm{~cm}$. This cell was only used in its full width, without including internal walls. The other two cells were made of glass plates. They had $h=(2.7 \pm 0.1) \mathrm{mm}, H=50 \mathrm{~cm}$, and $W_{c}=15 \mathrm{~cm}$, and $h=(2.85 \pm 0.05) \mathrm{mm}, H=30 \mathrm{~cm}$, and $W_{c}=21 \mathrm{~cm}$, respectively (the gap thickness of the latter cell could be modified and was also set to $h=(1.05 \pm 0.03) \mathrm{mm}$ for complementary investigations). In those cells, internal walls were included in order to reduce the effective width from $W_{c}$ to $W=9,7,4$, and $2.4 \mathrm{~cm}$. The motion of the bubble was recorded using a high-speed camera with a frame rate ranging between 150 and 1000 frames per second, and a spatial resolution between 100 and $200 \mu \mathrm{m} / \mathrm{px}$, depending 
on the experiments. A light-emitting diode backlight illumination was used to generate uniform lighting. The area, contour, and centroid of the bubble were obtained using typical image-processing techniques $[11,13]$. Quantitative measurements were performed in the upper region of the cell in which the bubbles had reached a stationary motion (i.e., after the transient following the bubble release).

In order to get an insight into the liquid dynamics associated with the changes in bubble motion due to the lateral confinement, a qualitative flow visualization approach was implemented in some experiments using spherical polystyrene particles with a diameter of $140 \mu \mathrm{m}$ and a density of $1.05 \mathrm{~g} / \mathrm{cm}^{3}$. Particles were seen as black spots with the same backlight illumination used to obtain the bubble path and were used as tracers of the flow.

The relevant quantities that remained fixed in the experiments are the densities $\left(\rho_{l}, \rho_{g}\right)$ and dynamic viscosities $\left(\mu_{l}, \mu_{g}\right)$ of the liquid and the gas phase, respectively, the surface tension $\sigma$, and the gravitational acceleration $g$. In addition, the thickness $h$ and the width $W$ of the cell and the bubble size $d$ were varied throughout the experiments. Six independent nondimensional control parameters can be formed from these quantities: the densities and viscosities ratios $\rho_{l} / \rho_{g} \gg 1$ and $\mu_{l} / \mu_{g} \gg 1$; two parameters characterizing the confinement of the bubble, $d / h$ and $d / W$, which throughout the experiments ranged between 1 and 14 and between 0.018 and 1.16, respectively; and the Archimedes number Ar and the Bond number Bo reading

$$
\mathrm{Ar}=\sqrt{g d} d / \nu \text { and } \mathrm{Bo}=\rho_{l} g d^{2} / \sigma,
$$

where $v=\mu_{l} / \rho_{l}$ is the liquid kinematic viscosity. In this work, we focused on bubbles whose size is larger than the cell gap $(d / h>1)$ and which are thus flattened between the cell plates. We explore a large range of bubble sizes ( $3 \mathrm{~mm}<d<40 \mathrm{~mm}$ ), corresponding typically to $500<\mathrm{Ar}<25000$ and $1.13<$ Bo $<215$.

Once the bubble velocity is known, the behavior of the bubble can be further characterized by two Reynolds numbers [20]:

$$
\operatorname{Re}=\frac{V_{b} d}{v} \quad \text { and } \quad \operatorname{Re}_{h}=\operatorname{Re}\left(\frac{h}{d}\right)^{2} .
$$

The first, Re, characterizes the in-plane motion of the bubble, and the second is associated with viscous diffusion in the gap. The inertial regime, which is the focus of the present work, corresponds to both $\operatorname{Re} \gg 1$ and $\operatorname{Re}_{h} \gg 1$.

\section{OVERVIEW OF THE DIFFERENT REGIMES}

We first present an overview of the different behaviors identified when the bubble size and the cell width vary. For each $W$ considered and $h \simeq 2.8 \mathrm{~mm}$, Fig. 2(a) shows the range of diameters $d$ associated with each behavior. The color of the data points is associated with the value of the cell width $W$ (empty black, $W=21 \mathrm{~cm}$; solid black, $W=15 \mathrm{~cm}$; orange, $W=9 \mathrm{~cm}$; blue, $W=7 \mathrm{~cm}$; green, $W=4 \mathrm{~cm}$; red, $W=2.4 \mathrm{~cm}$ ) and each symbol (being it empty or solid) represents a regime (denoted $I$ to $V I I$ ), as indicated in Fig. 2(b). This convention (colors and symbols) is used throughout the paper. An illustration of the different paths and shapes of the bubbles is provided in Fig. 2(b). The regimes match those reported in comparable conditions in the literature for particular cell widths $[12,16]$. However, a more refined classification is proposed here, presented as a function of the characteristic diameter $d$ of the bubble in the plane of the cell. Small bubbles $(d<1.3 \mathrm{~mm})$ follow a rectilinear path in the cell plane. For $1.3 \mathrm{~mm}<d<4 \mathrm{~mm}$, the bubbles display an oscillating path about the vertical direction and an ellipsoidal shape presenting oscillations of small amplitude (regime $I$, symbol $\diamond$ ). Note that, for most of these bubbles, $d$ is comparable to, or smaller than, the gap thickness $h$, so that their dynamics is governed by strong three-dimensional effects. The detailed investigation of such cases is beyond the scope of the present paper. As mentioned earlier, we here focus on bubbles displaying a two-dimensional behavior, with $d / h>1$. In the interval $4 \mathrm{~mm}<d<6 \mathrm{~mm}$, the shape oscillations increase in amplitude, and often a drift in the path of the 


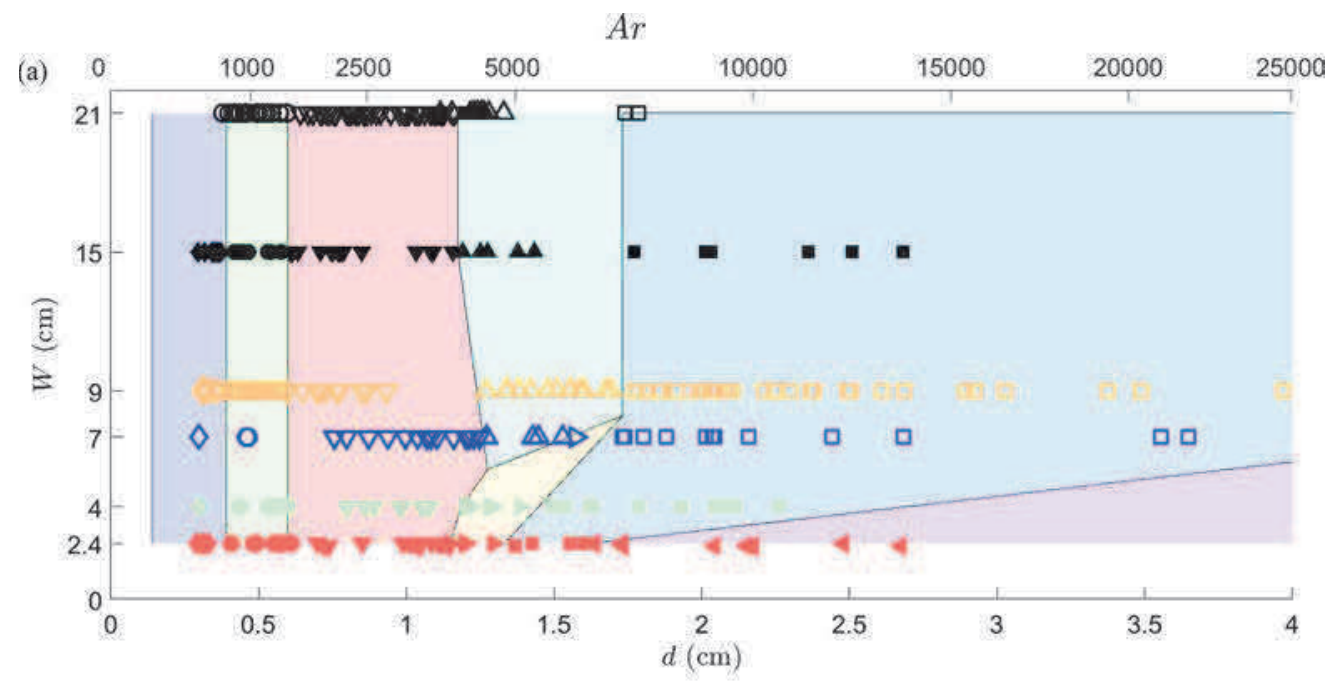

(b)

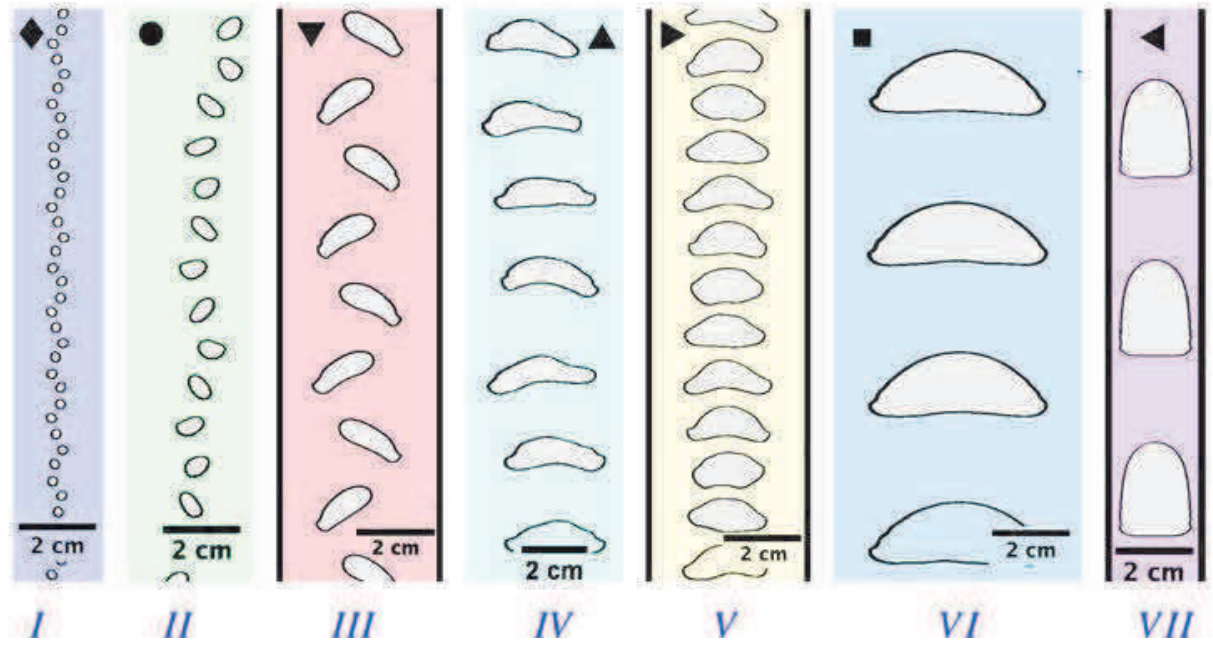

FIG. 2. (a) The different bubble behaviors observed in the $d-W$ plane for a cell with $h \simeq 2.8 \mathrm{~mm}$. The color of the data points is associated with the value of the cell width $W$ indicated by the vertical axis, and each symbol (being it empty or solid) represents a regime: $W=21 \mathrm{~cm}$, empty black; $W=15 \mathrm{~cm}$, solid black; $W=9 \mathrm{~cm}$, orange; $W=7 \mathrm{~cm}$, blue; $W=4 \mathrm{~cm}$, green; and $W=2.4 \mathrm{~cm}$, red. (b) Image superpositions illustrating the different regimes described in panel (a), which can be identified with the background color and the solid black symbol on the top. $\diamond$, path oscillations and weak shape oscillations about a vertical path; $\circ$, path oscillations and moderate shape oscillations (usually with a drift in the path); $\nabla$, regular large oscillations of both path and shape (about the vertical); $\triangle$, large and complex shape oscillations that induce irregular path deviations from the vertical; $\triangleright$, quasirectilinear path with periodic shape oscillations; $\square$, cylindrical-capped bubbles; and $\triangleleft$, slugs. The various regimes are denoted $I$ to $V I I$ as shown. The black vertical lines in regimes $I I I, V$, and $V I I$ represent the lateral walls.

bubble appears in addition to the path oscillation (regime $I I$, symbol $\circ$ ). Though the existence of a mean lateral drift of the path seems surprising, this feature has been reported for three-dimensional bubbles [21] (although with a smaller drift angle). For even larger bubble sizes $(d>6 \mathrm{~mm}$ and $d<$ $11.5-12.5 \mathrm{~mm}$, depending on the lateral confinement) regular path and shape oscillations remain, but the drift disappears (regime $I I I$, symbol $\nabla$ ). The largest bubbles studied in this work correspond 


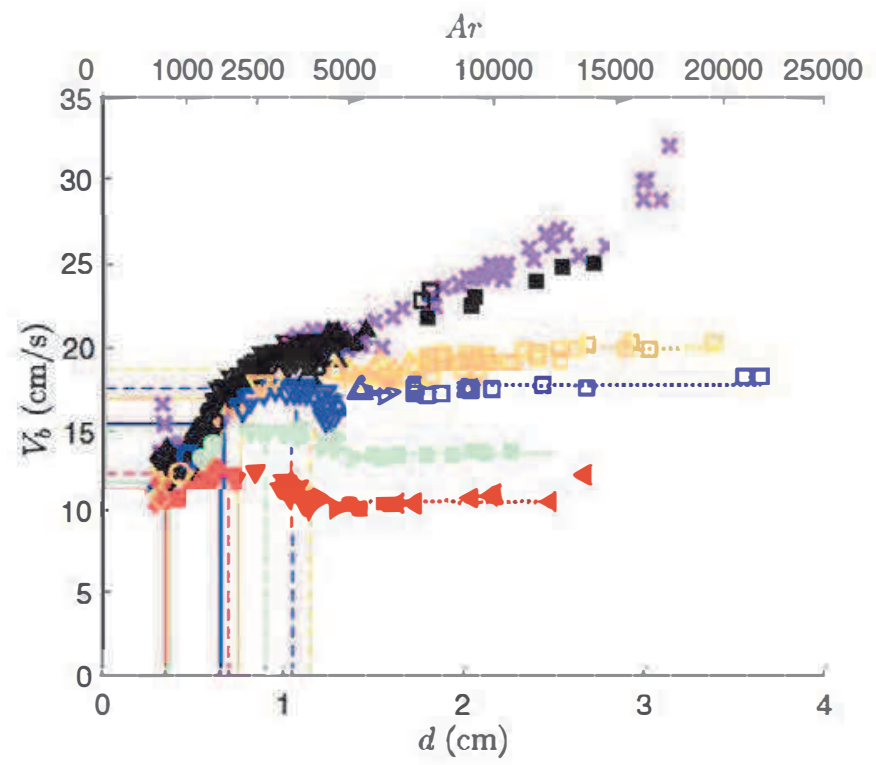

FIG. 3. Mean rise velocity of the bubble as a function of its planar diameter $d$ and of $\mathrm{Ar}$, for different cell widths $W(h \simeq 2.8 \mathrm{~mm}$ ). Same convention for colors and symbols as in Fig. 2(a). Magenta crosses correspond to the results from Filella et al. [12]. The dotted horizontal lines correspond to the constant velocity values used in Sec. VI for regimes $V I$ and $V I I$. For a given $W$, the critical and maximum diameters, $d_{c V}$ and $d_{m v}$, and the corresponding velocity values are indicated with solid and dashed lines, respectively.

to cylindrical-capped bubbles (regime $V I$, symbol $\square$ ) or slugs (regime $V I I$, symbol $\triangleleft$ ), for which no path or shape oscillations are observed. The path of the bubble is vertical and its shape is fixed, the top of the bubble being well described by an arc of a circle of radius $R_{c}$ [see Fig. 5(b)]. The slug regime is similar to the cylindrical-capped one but occurs for a greater lateral confinement (the bubble height being greater than $2 R_{c}$ ). The bubbles in these regimes have $d>17 \mathrm{~mm}$ for small lateral confinements, but they appear for smaller bubble sizes at greater confinements (e.g., for $d>$ $13 \mathrm{~mm}$ with $W=2.4 \mathrm{~cm}$ ). Between the regime of cylindrical-capped bubbles and the regime with regular path and shape oscillations, transitional regimes involving shape oscillations are observed (regimes $I V$ and $V$, symbols $\Delta$ and $D$ ). For smaller confinements, the shape oscillations induce irregular movements of the centroid (regime $I V$, symbol $\triangle$. On the other hand, for larger lateral confinements $(W \lesssim 7 \mathrm{~cm})$ the shape oscillations are periodic and stable, and the path of the centroid of the bubble is vertical (regime $V$, symbol $\triangleright$ ). For the intermediate case of $W=7 \mathrm{~cm}$, both regimes were observed (the stable one occurring for larger diameters).

\section{CHARACTERIZATION OF THE CONFINEMENT EFFECT ON THE BUBBLE BEHAVIOR}

\section{A. Impact on the mean rise velocity of the bubbles}

The mean rise velocity of the bubble, $V_{b}$, was determined by averaging its vertical velocity once the bubble had attained a stationary regime, and over an integer number of periods in the case of oscillating bubbles. The results are displayed in Fig. 3 as a function of the characteristic diameter $d$ of the bubble in the plane of the cell. For cell widths larger than $W=15 \mathrm{~cm}$ (black empty symbols), we obtained values of $V_{b}$ close to those for $W=40 \mathrm{~cm}$ (magenta crosses from Ref. [12]). Also, for all the $W$ values considered here, no effect of the cell width is visible in the vertical velocity for sufficiently small bubbles. However, for $W \leqslant 15 \mathrm{~cm}$, a departure of $V_{b}$ towards values smaller than those for larger $W$ occurs for a critical diameter, $d_{c V}$, that decreases with the cell width. Beyond 

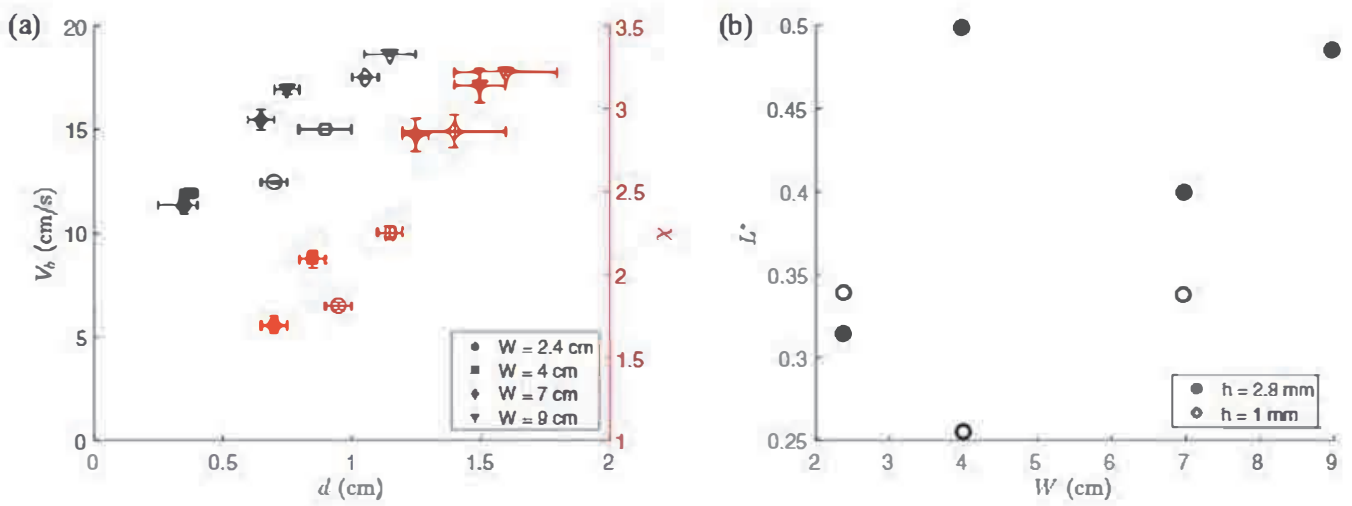

FIG. 4. (a) Diameters $d_{c v}$ (solid black), $d_{m v}$ (empty black), $d_{c \chi}$ (solid red), and $d_{m \chi}$ (empty red) along with the corresponding velocity and aspect ratio values, for different cell widths $W$ ( $h \simeq 2.8 \mathrm{~mm}$ ). (b) Values of $L^{*}$ corresponding to the critical diameters $d_{c v}$ for two gap thicknesses $(h \simeq 2.8$ and $1 \mathrm{~mm})$ and various $W$.

$d_{c V}$, for $W \leqslant 9 \mathrm{~cm}$, the mean rise velocity reaches a local maximum value (denoted $V_{b}\left(d_{m v}\right)$ for a diameter denoted $\left.d_{m v}\right)$ and eventually saturates at a constant value (smaller than $V_{b}\left(d_{m v}\right)$ for $W \leqslant 4 \mathrm{~cm}$ ). The constant velocity plateau is observed for cylindrical-capped bubbles (symbol $\square$ ) and slugs (symbol $\triangleleft$ ).

For each cell width $W$, the critical diameter $d_{c V}$ and the corresponding velocity $V_{b}\left(d_{c V}\right)$, as well as $d_{m v}$ and $V_{b}\left(d_{m v}\right)$, are indicated in Fig. 3 with solid and dashed lines, respectively. Their values are also displayed in Fig. 4(a) (black data points). Except for the smallest diameters (typically $<5 \mathrm{~mm}$ ) which correspond to oscillating ellipsoidal bubbles displaying almost no shape oscillation, $d_{c v}$ and $d_{m v}$ correspond to bubbles displaying both shape and path oscillations. For these oscillating bubbles, Filella et al. [12] showed that a strong dampening of the liquid velocity perturbation induced by the rising bubble occurs due to the interaction of the released vortices on a characteristic length scale of about $2 V_{b} / f, f$ being the path oscillation frequency of the bubble. The bubble can, therefore, be expected to become sensitive to the lateral confining walls when this length scale becomes comparable to the distance of the bubble to the wall, so that their ratio $L^{*}$ is expected to be of the order of unity,

$$
L^{*}=(W-d) f /\left(4 V_{h}\right) \sim 1
$$

The values of $L^{*}$ corresponding to the critical diameters $d_{c V}$ are shown in Fig. 4(b). Those obtained for a cell with a smaller gap $h \simeq 1 \mathrm{~mm}$ are also displayed to validate the analysis further, showing consistent results with $L^{*}$ values in the range of $0.3-0.5$ for both cases.

\section{B. Impact on the bubble shape}

We now turn our attention to the influence of the cell width $W$ on the bubble shape. This can be characterized by the aspect ratio $\chi=a / b$, defined as the average over the stationary regime of the ratio between the lengths of the major and minor axes ( $a$ and $b$, respectively) of the ellipse that best fits the bubble contour at each time step. Figure 5 illustrates the definition of $\chi$ by plotting the ellipse fitting the bubble contour for two characteristic bubble shapes, an oscillating bubble [panel (a)] and a cylindrical-capped bubble [panel (b)]. For consistency, in the case of slugs (regime VII, symbol $\triangleleft$ ), the inverse quantity is considered for $\chi$ as the bubble is longer than wider, leading to values of $\chi$ less than 1 in this regime. The evolution with $W$ of the aspect ratio $\chi$ characterizing the mean deformation of the bubble is presented in Fig. 6(a). Trends similar to those reported for $V_{b}$ can be identified: for sufficiently small bubbles or large enough cell widths, no effect of the confinement is observed. In the other cases, the lateral confinement results in a change of bubble shape, leading 
(a)

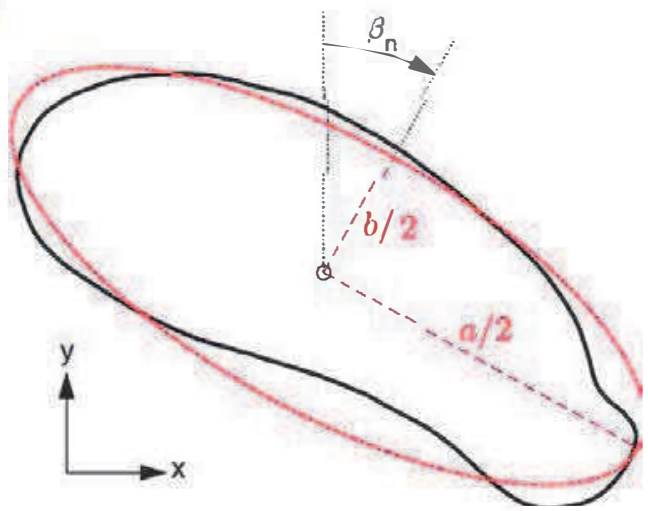

(b)

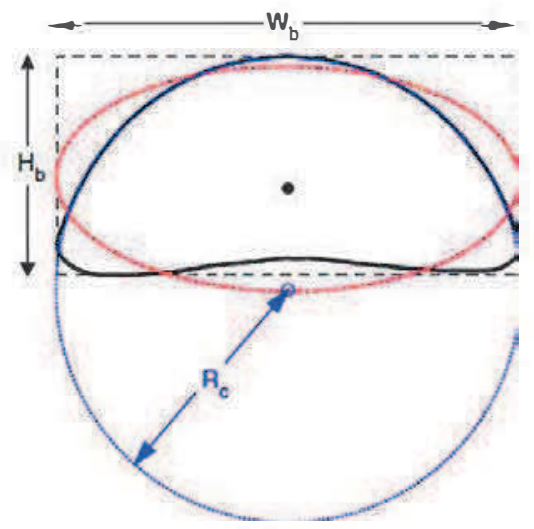

FIG. 5. Comparison of the bubble contour with the ellipse used to characterize its shape for (a) an oscillating bubble and (b) a cylindrical-capped bubble. In the latter case, we also show additional characteristic dimensions of the bubble that are used in Sec. VI: the radius of curvature at the bubble front, $R_{c}$, and the height $H_{b}$ and the width $W_{b}$ of the bubble.

to shapes closer to circular ( $\chi$ closer to 1$)$. For given $W$, the departure of $\chi$ from the behavior in the unconfined configuration ( $W=40 \mathrm{~cm}$, magenta crosses) occurs, however, at a critical diameter $d_{c \chi}$ larger than $d_{c v}$. The values of $d_{c \chi}$ (and of $\chi\left(d_{c \chi}\right)$ ) obtained for each $W$ are plotted in Fig. 4(a) (red data points) for comparison with those of $d_{c v}$, showing that the effect of confinement on bubble deformation is visible only for larger diameters, about twice those for which the rise velocity starts to be modified. Figure 4(a) also provides the maximum values $\chi\left(d_{m_{X}}\right)$ of the aspect ratio $\chi$ along with the corresponding diameters $d_{m \chi}$, characterizing the maximal deformation experienced by the bubbles for a given cell width $W$. Both diameters, $d_{c x}$ and $d_{m_{X}}$, correspond to bubbles displaying shape oscillations. Finally, it can be pointed out that the evolutions of $\chi$ displayed in Fig. 6(a) appear similar for all $W$. To highlight the generic character of the evolution of the aspect ratio, we plot in Fig. 6 (b) the aspect ratio $\chi$ compared to the parameter characterizing the in-plane confinement of
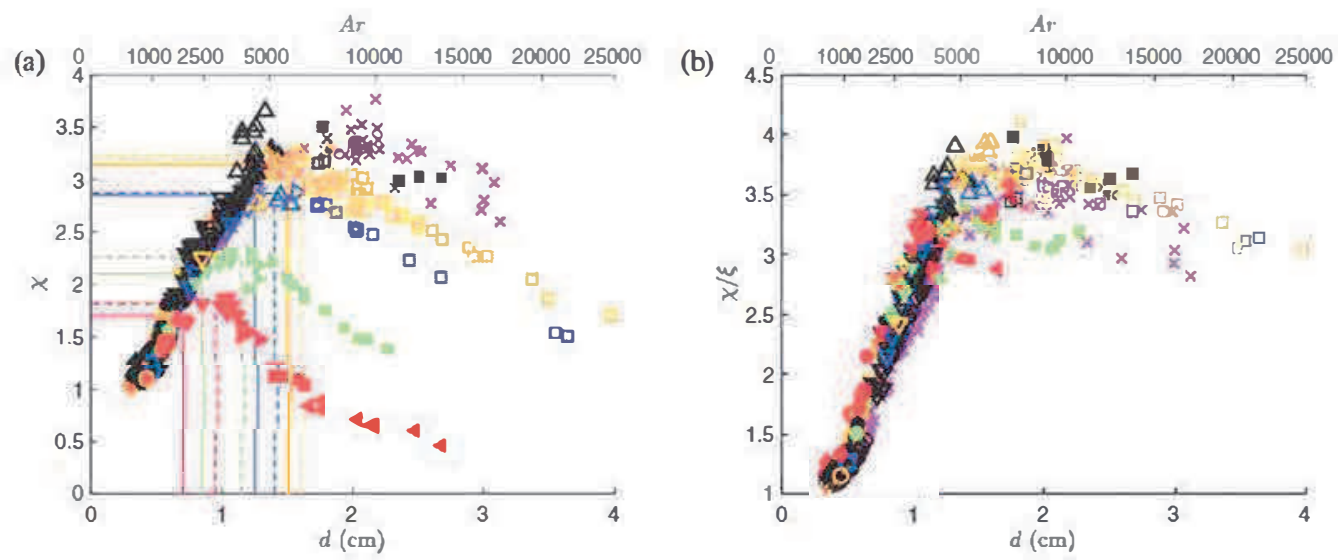

FIG. 6. (a) Mean aspect ratio of the bubble as a function of the planar diameter of the bubble $d$ and of the Archimedes number Ar, for different cell widths $W(h \simeq 2.8 \mathrm{~mm})$. For a given $W$, the critical and maximum diameters, $d_{c \chi}$ and $d_{m \chi}$, and the corresponding $\chi$ values are indicated with solid and dashed lines, respectively. (b) Mean aspect ratio $\chi$ compared to the normalized distance left between the bubble and the wall $\xi$ for different Ar and $W$. Same convention as in Fig. 2(a). 
(a)

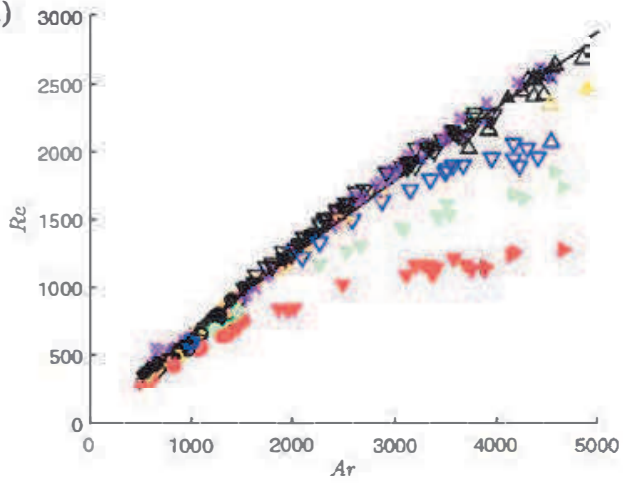

(b) 3500

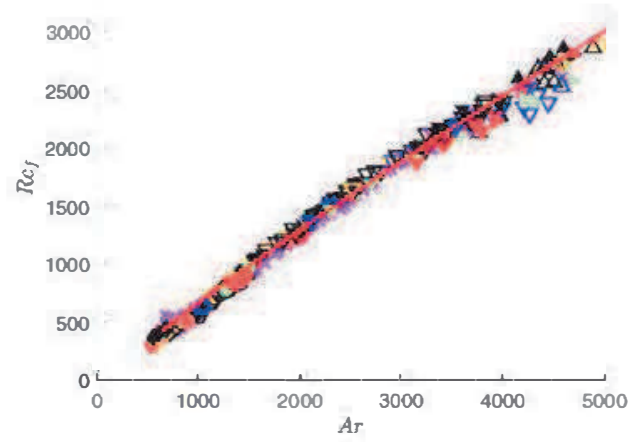

FIG. 7. (a) $\operatorname{Re}=V_{b} d / v$ and (b) $\operatorname{Re}_{f}=\operatorname{Re} / \xi$ characterizing the intensity of the counterflow generated by a rising bubble for different cell widths $W(\boldsymbol{h} \simeq 2.8 \mathrm{~mm}$ ), as functions of the Archimedes number of the bubble. Same convention as in Fig. 2(a). The solid black line in panel (a) corresponds to the law (1) from Filella et al. [12], and the solid red line in panel (b) corresponds to expression (10).

the bubble,

$$
\xi=1-d / W
$$

showing a single trend in the regimes $I$ to $V$ for all the cell widths investigated.

We have seen that when wall effects become significant, a greater confinement (smaller $W$ for a fixed bubble size) leads to more circular shapes as well as to lower rise velocities of the bubbles. To gain a deeper understanding of the effect of the transverse confinement on the bubble behavior, we now investigate separately the two salient regimes identified: the case of oscillating bubbles, for which the departure from the behavior in the unconfined situation becomes apparent (Sec. V), and the case of large bubbles, for which the mean rise velocity saturates at a constant value, independent of the bubble volume (Sec. VI).

\section{THE REGIME OF OSCILLATING BUBBLES}

A. Scaling law for the mean rise velocity of oscillating bubbles

We focus here on bubbles displaying path oscillations in regimes $l$ to $I I I$ (symbols $\diamond, \circ$, and $\nabla$ ). These correspond to the first three types of oscillatory motion displayed in Fig. 2(b), typically observed for $500 \lesssim A r \lesssim 5000$ for $W \geqslant 9 \mathrm{~cm}$ [Fig. 2(a)]. For these bubbles, the evolution of the Reynolds number, $\operatorname{Re}=V_{b} d / \nu$, with the Archimedes number is shown in Fig. 7(a) for different cell widths $W$. The experimental results for a cell of large width ( $W=40 \mathrm{~cm}$, magenta crosses) and the curve (black solid line) corresponding to relation (1) obtained by Filella et al. [12] are also superposed on the results. As discussed in the previous section, when the cell width decreases, an earlier departure from the unconfined evolution occurs, as well as a decrease in the mean rise velocity for a bubble of given diameter (or Ar). To get an insight into this behavior, we consider how the liquid flow surrounding the bubble adapts to the confining walls. As the lateral walls impact the bubble motion through the no-slip condition imposed on the flow generated by the bubble, we assume that the mean rise velocity of the bubble is related to the control parameter $\xi=1-d / W$, comparing the space let free for the downward motion of the liquid with the total width of the cell. The upward motion of the bubble induces a downward motion of the liquid, which, to satisfy mass conservation, can be estimated as a first approximation by the mean downward velocity,

$$
U_{f}=V_{b} \frac{d}{\mathrm{WI} \quad d}
$$


A relative velocity between the bubble and the liquid can then be introduced as

$$
U_{\text {rel }}=V_{b}+U_{f}=V_{b} \frac{W}{W-d}=\frac{V_{b}}{\xi} .
$$

This velocity can be used to build the Reynolds number

$$
\operatorname{Re}_{f}=\frac{U_{\mathrm{rel}} d}{v}=\frac{\operatorname{Re}}{\xi}
$$

accounting for the intensity of the counterflow generated by the rising bubble in the cell. Note that when the confinement vanishes, $d / W \rightarrow 0$ and $\operatorname{Re}_{f} \rightarrow$ Re. Figure 7(b) presents the evolution of $\operatorname{Re}_{f}$ with the Archimedes number of the bubble for different cell widths $W$. We can observe that the values for $\mathrm{Re}_{f}$ lie on a single master curve that depends on Ar but no longer depends on the cell width $W$ and that matches the evolution in the unconfined situation (large $W$ ). For all the cell widths investigated, this result indicates that, for a given bubble size, the bubble velocity is adjusted according to the space let free for the passage of the flow induced by the bubble. Furthermore, the master curve follows closely the law (1) proposed by Filella et al. [12], leading in this crude analysis to $\operatorname{Re}_{f}$ independent of $W$ and $U_{\text {rel }} \simeq V_{b, \infty}$ at given Ar for all $W$. However, a slight correction in the constant prefactor $k$ of expression (1) is necessary to account for the effect of $\xi$ even at large $W$. We find that the bubble velocity $V_{b}$ can be estimated by

$$
V_{b} \simeq k \xi\left(\frac{h}{d}\right)^{1 / 6} \sqrt{g d}, \quad \text { with } k=0.8 .
$$

This relation is shown with a red line in Fig. 7(b), showing a very good agreement with experimental data. Expression (10), therefore, generalizes the law (1) valid for laterally unconfined bubbles $(W=$ $40 \mathrm{~cm}$ ) to the case of bubbles in the presence of in-plane confinement. Note that we also incorporated in both Figs. 7(a) and 7(b) the data points corresponding to the transitional regimes $I V$ and $V$ (symbols $\triangle$ and $\triangleright$, respectively) and that the analysis acceptably holds also for these cases. From this expression for the velocity, an expression for the drag coefficient including the effect of the walls can be derived and is presented in Appendix A.

\section{B. Scaling law for the mean aspect ratio of oscillating bubbles}

As seen in Sec. IV, at issue is also the impact of the transverse confinement on the bubble shape. For a cell with large $W$, Filella et al. [12] showed that the Weber number, We $=\rho_{l} V_{b}^{2} d / \sigma$, determines at leading order the mean deformation of the bubble (characterized by the mean aspect ratio $\chi$ ) independently of the gap thickness $h$. They observed values of $\chi$ close to those determined by Roig et al. [11] for the same We and the same couple of fluids, although the diameters of the bubbles, their volumes, and their velocities were different. They further proposed the relationship

$$
\chi \simeq 1.12 \sqrt{\mathrm{We}}
$$

for the mean aspect ratio of the bubble as a function of the Weber number in the range $1<\mathrm{We}<10$. For the different cell widths considered here, the evolution of $\chi$ with $\sqrt{\mathrm{We}}$ is displayed in Fig. 8 . The superposition of the different curves indicates that, at leading order, the same balance between inertia and surface tension effects governs the bubble shape, for the different cell widths $W$.

\section{Scaling laws for the bubble oscillatory motion}

We now proceed with the investigation of the wall effect on the characteristics of the oscillatory motion of the bubbles. In this regime, the path of the bubbles can be characterized with quantities (degrees of freedom in translation and rotation) evolving periodically in time, as discussed in Roig et al. [11] and Filella et al. [12] for large $W$. For each quantity, the amplitude of oscillation is obtained from a sinusoidal fit of the experimental signal. Oscillations about the mean rise velocity 


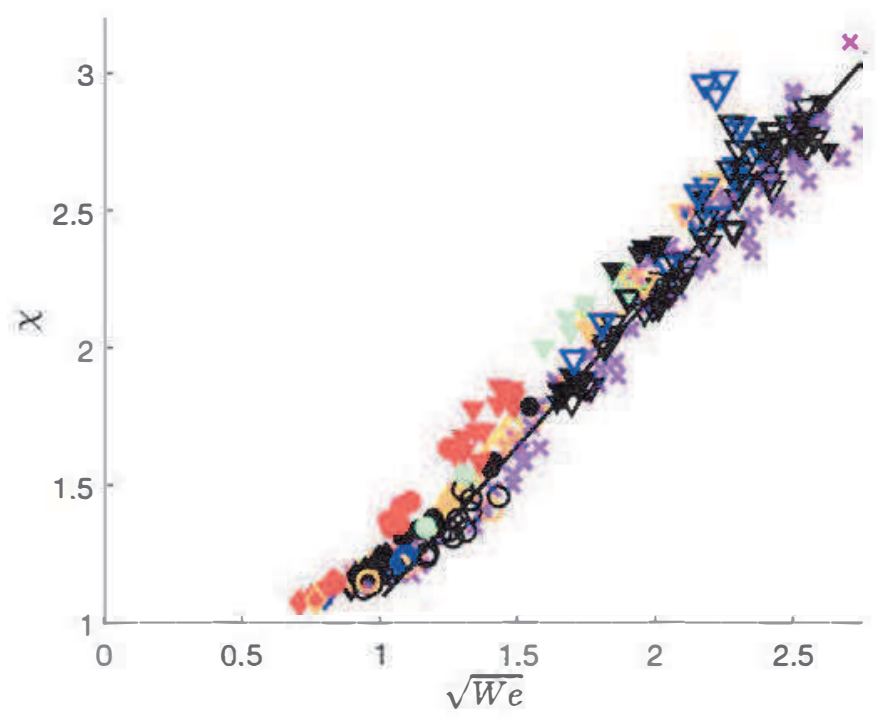

FIG. 8. Aspect ratio $\chi$ of oscillating bubbles as a function of $\sqrt{W e}$ for different cell widths $W$ ( $h \simeq 2.8 \mathrm{~mm}$ ). Same convention as in Fig. 2(a). The solid black line corresponds to the expression $\chi \simeq 1.12 \sqrt{\text { We }}$ from Filella et al. [12].

$V_{b}$ take place in the vertical direction at a frequency of $2 f$ with an amplitude, denoted $\widetilde{V}_{y}$. This has the same dependence on Ar for all the cell widths investigated, a property shared with the relative velocity $U_{\text {rel }} \simeq V_{b, \infty}$, as confirmed from Fig. 9(a). As regards the horizontal oscillatory motion at the frequency $f$, the amplitude of the horizontal displacement $\left(\widetilde{A_{x}}\right)$ is displayed in Fig. 9(b). For most of the cell widths considered, the amplitude $\widetilde{A}_{x}$ is not significantly affected by the confinement, being at leading order related to the bubble size $d$. For weaker confinements, $\widetilde{A}_{x}$ corresponds approximately to half a diameter $d$, while it becomes increasingly smaller for sufficiently large and confined bubbles (typically, $W \leqslant 4 \mathrm{~cm}$ and $d / W>0.2$ ). We will come back to these cases later. The corresponding amplitudes of the horizontal velocities, $\widetilde{V}_{x}$, show a comparable behavior when normalized with $U_{\text {rel, }}$ with a typical value of 0.5 for $\mathrm{Ar} \simeq 2500$ [the results are not explicitly shown

(a)

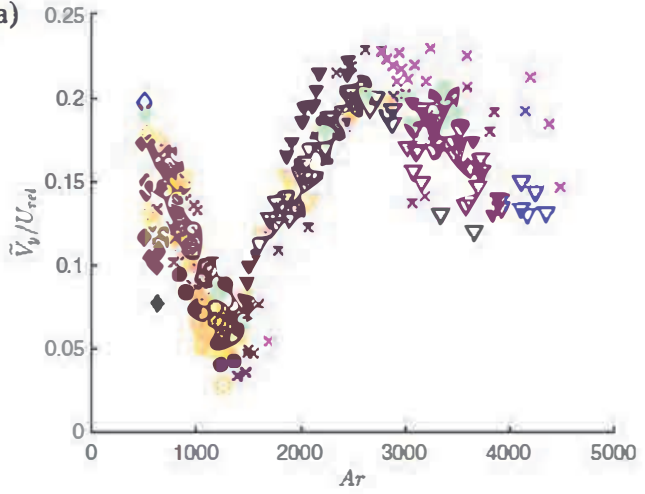

(b)

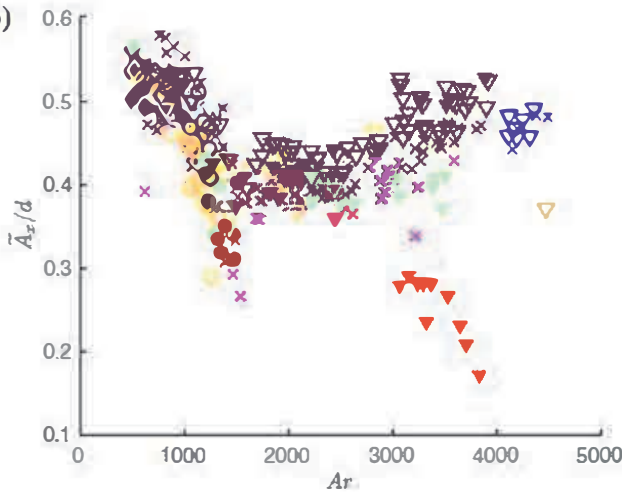

FIG. 9. Translational components of the bubble oscillatory motion: (a) Vertical velocity amplitude normalized with the relative velocity $U_{\text {rel }}$ and (b) amplitude of horizontal displacement normalized with $d$, as functions of the Archimedes number, for different cell widths $W(h \simeq 2.8 \mathrm{~mm})$. Same convention as in Fig. 2(a). Magenta crosses correspond to the results from Filella et al. [12] for an unconfined cell $(W=40 \mathrm{~cm})$. 

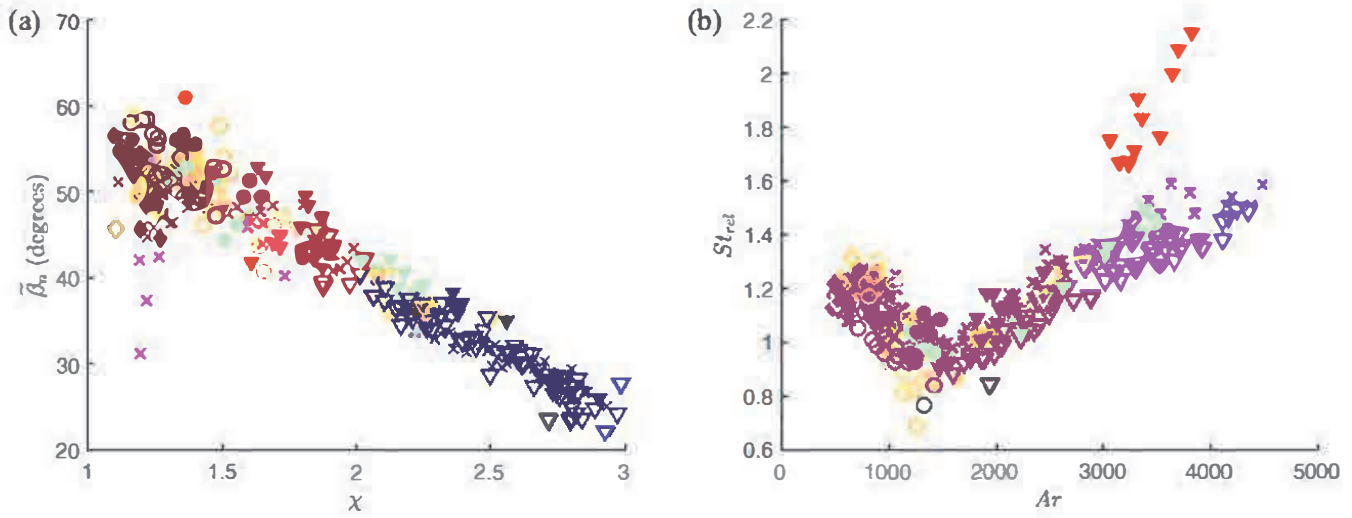

FIG. 10. (a) Amplitude of the inclination angle $\widetilde{\beta}_{n}$ of the bubble as a function of its aspect ratio $\chi$, for different cell widths $W\left(h \simeq 2.8 \mathrm{~mm}\right.$ ). (b) Strouhal number, $\mathrm{St}_{\text {sel }}$, based on the frequency of path oscillation and on the relative velocity $U_{\text {ret }}$ as a function of the Archimedes number of the bubble, for different cell widths $W(h \simeq 2.8 \mathrm{~mm})$. Same convention as in Fig. 2(a). In both figures, magenta crosses correspond to the values $\widetilde{\beta}_{n, \infty}$ and $\mathrm{St}_{\infty}$ reported in Filella et al. [12] for an unconfined cell $(W=40 \mathrm{~cm})$.

here, but can be simply obtained by multiplying the values of Fig. 9(b) with those of Fig. 10(b)]. Note also in Figs. 9(a) and 9(b) the changes in the evolutions with Ar for both translational components. These correspond essentially to the regimes $I, I I$, and $I I I$ of path and shape oscillations (symbols $\diamond, \circ$, and $\nabla$ ) shown in Fig. 2(b).

In the regime of both path and shape oscillations, Filella et al. [12] highlighted the crucial role of the mean deformation of the bubble $\chi$ on the bubble kinematics. Following this idea and as $\chi$ is also impacted by the confinement (Fig. 6), we investigated how the inclination angle of the bubble is affected by the confinement and related to the bubble mean deformation. The inclination angle of the bubble $\beta_{n}$ is defined as the angle between the vertical direction and the minor axis of the ellipse fitting its contour, as indicated in Fig. 5(a). Figure 10(a) plots the amplitude of oscillation $\widetilde{\beta}_{n}$ of the inclination angle obtained as a function of the bubble mean aspect ratio $\chi$. We see that these two quantities are strongly linked, as their relationship is not affected by variations in the bubble velocity (i.e., $V_{b}$ ) or the cell width $W$. For given $\mathrm{Ar}$, as the cell width decreases, $\chi$ also decreases and larger inclinations of the bubble occur accordingly.

We also determined in this regime the frequency $f$ of path oscillations for various confinement widths $W$. The values used here were obtained from the sinusoidal fits of the $V_{x}$ signals for each bubble (differences with the values of $f$ provided by the other quantities are less than $2 \%$ ). We observed that $f$ is not significantly affected by the transverse confinement, except for sufficiently large and confined bubbles (typically $d / W>0.4$ obtained for $W=2.4 \mathrm{~cm}$ ), which exhibit higher oscillation frequencies in association with the lower amplitudes of oscillation observed in Fig. 9. The combination of these two features, which indicate a strong effect of the walls on the bubble motion when $\xi<0.6$, can be interpreted as a "distant bouncing" behavior of the bubble on the lateral walls, the bubble being repulsed back and forth from one wall to the other during its rise due to the intense downward flow it generates when it deviates from the middle of the cell. Except for these bubbles, frequency selection can be associated with the inertial time scale $d / U_{\text {rel }}$, corresponding to a Strouhal number, $\mathrm{St}_{\text {rel }}=2 \pi f d / U_{\text {rel }} \simeq \mathrm{St}_{\infty}$, independent of $W$, as can be seen in Fig. 10(b). Conversely, as the mean rise velocity of the bubble $V_{b}$ decreases with $W$, the spatial wavelength associated with the bubble oscillation, $V_{b} / f$, decreases with $W$. The consequence of this result is that a larger number of vortices are produced by the oscillating bubble over a given spatial distance, when the transverse confinement becomes stronger. The amount of vortices produced by a bubble of given volume in a given time interval is instead the same whatever the confinement width. This remarkable 
(a)

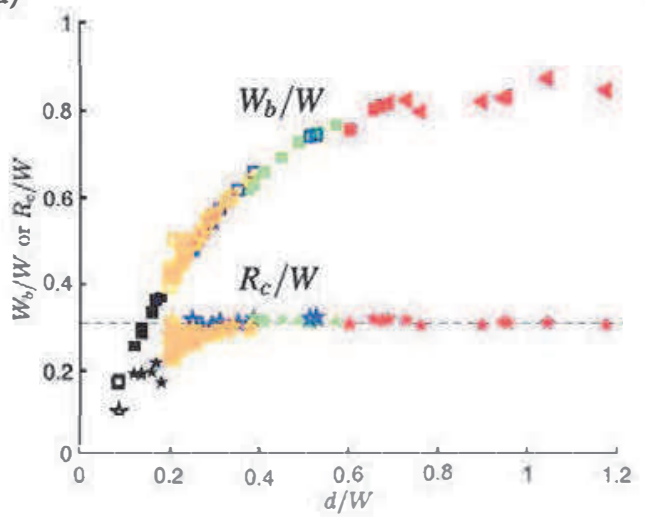

(b)

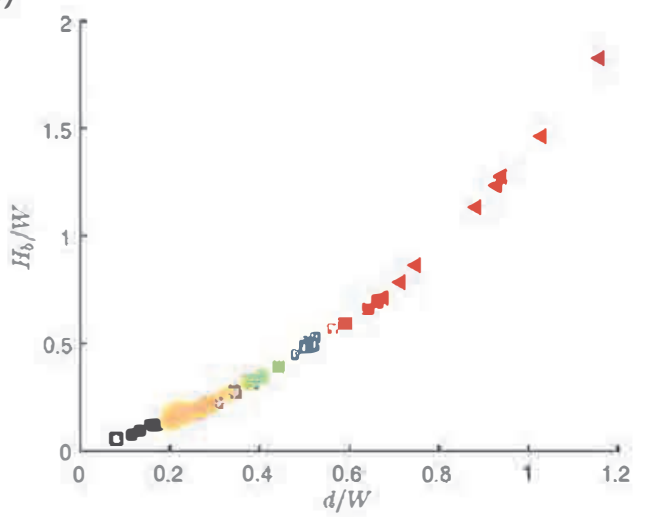

FIG. 11. Evolutions of (a) $W_{b} / W$ (upper curve), $R_{c} / W$ (lower curve), and (b) $H_{b} / W$, describing the shape of confined cylindrical-capped bubbles, when their volume increases $(h \simeq 2.8 \mathrm{~mm})$. The horizontal dashed line in panel (a) corresponds to $R_{c} \simeq 0.31 \mathrm{~W}$. Same convention as in Fig. 2(a) (except for $R_{c}$, where stars are used as symbols).

feature indicates that the confinement has an important impact on the vortex street generated by the oscillating bubble. A visualization of this effect is provided in Appendix B.

\section{THE LIMIT OF LARGE BUBBLES}

A. Shape properties of cylindrical-capped bubbles

We now consider larger bubbles, corresponding to cylindrical-capped bubbles displaying a stationary shape and rectilinear motion. The most representative regime for these bubbles is regime $V I$ (symbol $\square$ ). However, for sufficiently strong confinements (here for $W=2.4 \mathrm{~cm}$ ), slugs emerge (regime $V I I$, symbol $\triangleleft$ ), the bubble extending essentially in length when its volume is increased. For both regimes $V I$ and $V I I$, a description of the bubble shape can be obtained by introducing the radius of curvature at the bubble front $R_{c}$, as well as the width $W_{b}$ and the height $H_{b}$ of the bubble, defined in Fig. 5(b). Interestingly, as can be seen in this figure, the aspect ratio $\chi$ provides a very good approximation to the ratio $W_{b} / H_{b}$ in regime $V I(\square)$. The difference between these two quantities was observed to be less than 5\% in most cases. Figures 11(a) and 11(b) display the evolutions of the bubble width $W_{b}$ and height $H_{b}$, providing for both quantities a single trend as the bubble size increases and the cell width $W$ changes. We can also observe that the width increases faster than the height, but eventually saturates due to the transverse confinement (corresponding to the emergence of slugs), whereas the height continues to increase. We also determined the radius of curvature at the bubble front, $R_{c}$, for the different cell widths. The results are displayed in Fig. 11(a) (star symbols) indicating that for sufficiently confined bubbles, $d / W>0.2(\xi<0.8)$ for $h \simeq 2.8 \mathrm{~mm}, R_{c}$ depends only on $W$ through the simple relation

$$
R_{c} \simeq 0.31 W \text {. }
$$

Beyond this threshold, as the bubble volume increases, the shape of the bubble is unaffected at its front, and the bubble simply extends in length and width (or only in length for slugs). This feature is illustrated in Fig. 12(a) for various cell widths $W$, showing that $R_{c}$ remains unchanged when the bubble size, characterized by the Archimedes number Ar, increases. The superposition of the bubble contours with dimensions renormalized with $W$, presented in Fig. 12(b), shows further that the bubble shapes are identical for the different cell widths. As the relation $R_{c} \simeq 0.31 W$ also holds for the experiments performed by Collins [18] with $h \simeq 6.35 \mathrm{~mm}$, we performed equivalent measurements in a cell of smaller gap thickness $h \simeq 1 \mathrm{~mm}$ for $W=2.4,4$, and $7 \mathrm{~cm}$ to further 
(a)

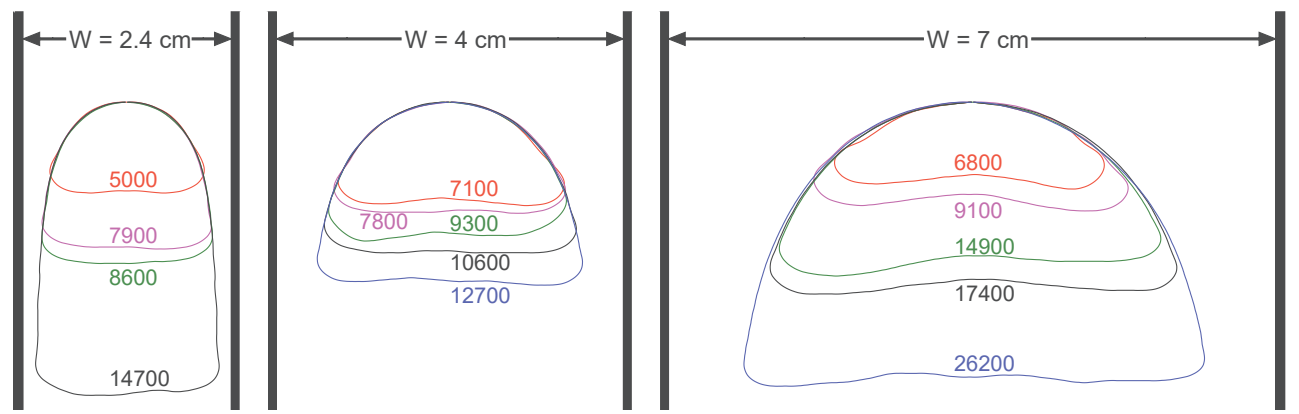

(b)
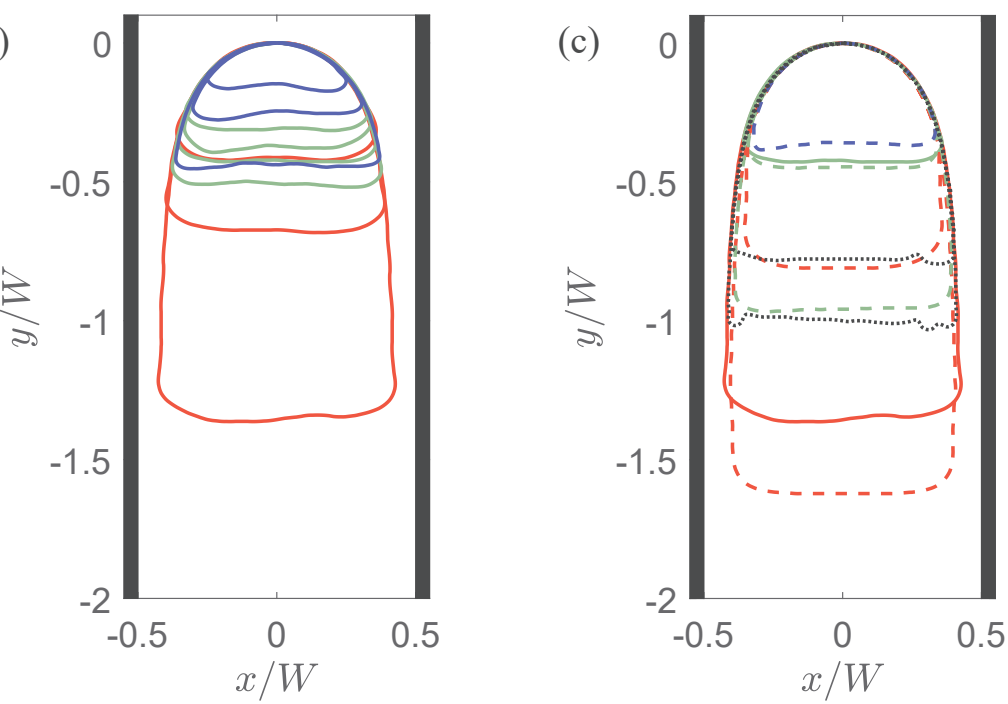

FIG. 12. (a) Illustration of the shape evolution of confined cylindrical-capped bubbles, when their volume is increased, showing the invariance of the radius of curvature at the bubble front. The position of the channel walls are shown with thick black lines, and three channel widths $W$ are presented. The colored numbers indicate the Archimedes number of the corresponding bubble contour $(h \simeq 2.8 \mathrm{~mm})$. (b) and (c) Superposition of the bubble contours for different $W$ and gap thicknesses $h$ : blue, $W=7 \mathrm{~cm}$; green, $W=4 \mathrm{~cm}$; red, $W=2.4 \mathrm{~cm}$; solid bubble contours, $h \simeq 2.8 \mathrm{~mm}$; dashed contours, $h \simeq 1 \mathrm{~mm}$; black dotted contours, $h=6.35 \mathrm{~mm}$ and $W=7.9 \mathrm{~cm}$ from Collins [18].

explore the effect of the confined cell geometry on the bubble shape. In this latter configuration, we obtained that relation (12) is verified when $d / W>0.3(\xi<0.7)$. Figure 12(c) illustrates the genericity of relation (12) by comparing renormalized bubble shapes obtained for cells with different $W$ and $h$. No effect of the gap size is visible on the bubble front or on the evolution of the interface next to the walls. Slight differences can only be observed at the rear of the bubble, in particular on $W_{b}$ and on the curvatures at the right and left round corners.

\section{B. Velocity of cylindrical-capped bubbles}

From the derivation of the two-dimensional potential flow about a confined cylindrical-capped bubble, Collins [18] showed that the rise velocity of the bubble is related to the radius of curvature at the bubble's front, being proportional to $\sqrt{g R_{c}}$. This result also holds here for all the bubbles in regimes $V I$ and $V I I$ for $W \leqslant 9 \mathrm{~cm}$. As also indicated by Collins [18] and observed here for 
(a)

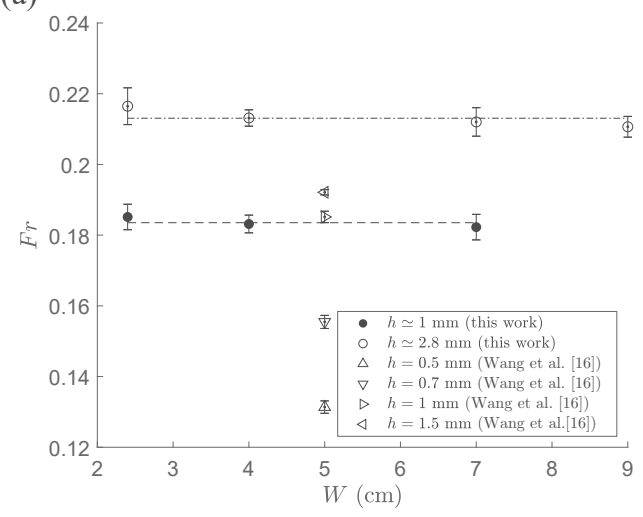

(b)

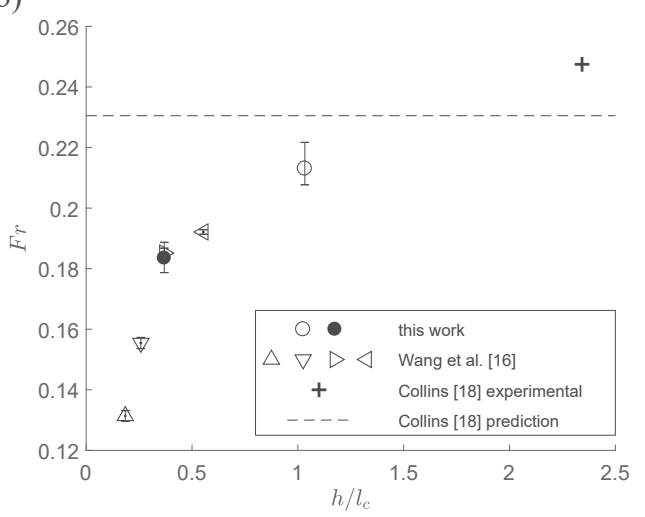

FIG. 13. (a) Froude number as a function of the cell width obtained in different experiments for different gap thicknesses. The data points from this work and those from Ref. [16] correspond to averages over different sizes of cylindrical-capped bubbles. The horizontal lines correspond to the average values for Fr over several cell widths. (b) Froude number observed for cylindrical-capped bubbles as a function of the gap thickness $h$ normalized with the capillary length $l_{c}=\sqrt{\sigma /\left(\rho_{l} g\right)} \simeq 2.7 \mathrm{~mm}$. The results from this work correspond to the average over several cell widths.

sufficiently confined bubbles verifying relation (12), the mean rise velocity of the bubbles remains then constant for a given confinement $W$ independent of their size $d$ (Fig. 3), and proportional to $\sqrt{g W}$. Denoting Fr the Froude number,

$$
\operatorname{Fr}=V_{b} / \sqrt{g W}
$$

our experiments provide values for Fr in the range $0.210-0.217$ for the various cell widths $W$ investigated and for $h \simeq 2.8 \mathrm{~mm}$. Differences in the values for the Froude number are, however, found in the literature. Birkoff and Carter [22] obtained $\mathrm{Fr}=0.29$ for a gap thickness of $2.54 \mathrm{~cm}$ (1 in.) and $W=10.3 \mathrm{~cm}$. For a cell with a gap thickness of $h=6.35 \mathrm{~mm}$ and $W / h>10$, Collins [18] reported an experimental determination for Fr higher by approximately $9 \%$ than their own theoretical prediction, $\operatorname{Fr}=(6 \pi)^{-1 / 2} \simeq 0.23$, a difference they attributed to the three-dimensional character of the flow near the stagnation point at the bubble's front. Wang et al. [16] measured the rise velocities of cylindrical-capped bubbles in cells with lower gap thicknesses (ranging from 0.5 to $1.5 \mathrm{~mm}$ ) and $W$ fixed to $5 \mathrm{~cm}$. Even though values for the Froude number were not reported in their work, they can be obtained from their results, yielding also contrasted results. This led us to investigate the effect of the gap thickness on Fr, by measuring the rise velocities of bubbles in a cell of smaller gap thickness $(h \simeq 1 \mathrm{~mm}$ for $W=2.4,4$, and $7 \mathrm{~cm}$ ), which provided values for Fr in the range of $0.182-0.185$ for the same relation $R_{c} \simeq 0.31 W$. Figure 13 (a) summarizes the results obtained for Fr in this work for $h \simeq 2.8 \mathrm{~mm}$ and $h \simeq 1 \mathrm{~mm}$ and different cell widths $W$, and it summarizes the results from Wang et al. [16] for the same couple of fluids in cells having various gap thicknesses. Figure 13(b) further shows the values for Fr plotted as a function of the gap thickness normalized with the capillary length $l_{c}=\sqrt{\sigma /\left(\rho_{l} g\right)}$. The effect of the gap thickness is conspicuous in both figures, the Froude number diminishing with $h$. Furthermore, the decrease rate becomes stronger for $h / l_{c} \leqslant 0.5$, beyond which surface tension effects can be, in fact, expected to affect more significantly the pressure inside the bubble. These results highlight the differences with bubbles of comparable shape in axisymmetrical configurations (see, for instance, Ref. [23]) or in channel geometries with dimensions larger than $l_{c}$ (see, for instance, Refs. [22,24] and references therein). 


\section{CONCLUDING REMARKS}

We investigated the effect of the transverse width $W$ of the cell on the behavior of bubbles freely rising in a thin-gap cell at high Reynolds numbers. Beyond a critical bubble size $d$ (or Archimedes number Ar), which depends on the cell width $W$, the behavior of the bubble adjusts to the presence of the confinement. This leads to a lower mean rise velocity of the bubble, $V_{b}$, and to a modification of its shape, which becomes closer to circular. Both effects become stronger as the cell width decreases.

We first focused our attention on oscillating bubbles (occurring typically for $500<\mathrm{Ar} \leqslant 5000$ ), for which the departure from the behavior in the unconfined situation (large $W$ ) occurs. We introduced the relative velocity $U_{\text {rel }}$ accounting for the variable strength of the downward flow generated by the bubble when the confinement changes, and we found $U_{\text {rel }}=V_{b} / \xi \simeq V_{b, \infty}$ for all the cell widths considered, with $\xi=1-d / W$. This allowed us to emphasize the role of the confinement ratio $\xi$ and to obtain a simple expression estimating at leading order the velocity of the bubble, $V_{b} \simeq 0.8 \xi\left(\frac{h}{d}\right)^{1 / 6} \sqrt{g d}$. We also determined the corresponding expression for the drag coefficient experienced by the bubble. We further investigated how the oscillatory motion of the bubble is impacted by the confinement. We showed that, for a given diameter $d$ and confinement ratio $\xi$, the translational and rotational components characterizing the bubble oscillatory motion (frequency and amplitudes) can be predicted using the characteristic length and velocity scales, $d$ and $U_{\text {rel }}$. We also highlighted the strong coupling that exists between the mean deformation of the bubble and its inclination angle. Finally, we showed that, while the frequency of oscillation of the bubble is not significantly affected by the presence of the walls, the spatial wavelength of the path becomes shorter when $W$ decreases, resulting in the production of smaller and more numerous vortices in the cell. It is interesting to point out that these results are reminiscent of those encountered when the bubble freely rises in a moderate counterflow [25].

We then considered the case of cylindrical-capped bubbles and slugs, and we showed that the shape of the bubble in the plane of the cell is essentially governed by the channel width $W$, independently of the gap thickness and of the bubble velocity. For a large range of bubble sizes and cell widths, we confirmed the saturation of the bubble vertical velocity to a constant value for a given $W$, independent of the bubble size. This regime is characterized by a constant Froude number, $\mathrm{Fr}=V_{b} / \sqrt{g W}$, that is shown to strongly depend on the gap thickness of the cell. The dependence on $W$ of the radius of curvature at the bubble's front was also emphasized in association with this velocity scaling.

\section{ACKNOWLEDGMENTS}

The authors are grateful to G. Ehses, G. Albert, and L. Mouneix for building the IMFT experimental setup and to S. Cazin for his help with the optical technique. This collaboration was possible thanks to the financial support from CONICET and Universidad de Buenos Aires, Argentina, and from INPT, Toulouse (SMI fundings 2016 and 2018), and it became part of the IRP CNRS-CONICET IVMF in 2019.

\section{APPENDIX A: DRAG COEFFICIENT FOR OSCILLATING BUBBLES}

The dependence of the rise velocities of the bubbles with $\xi$, shown in Fig. 7 and formalized with expression (10) for oscillating bubbles, indicates that the presence of the lateral confinement causes an additional drag on the bubble. Following Roig et al. [11], and neglecting friction in the thin liquid films separating the bubble and the gap walls (shear-free boundary condition), the mean balance of the vertical forces (drag force $D$ and buoyancy) acting on a bubble rising in a thin-gap cell of lateral width $W$ can be written as

$$
D=\frac{1}{2} \rho_{l} \mathscr{S}_{W} C_{D, W} V_{b}^{2}=\left(\rho_{l}-\rho_{g}\right) g \mathscr{V}
$$


(a)

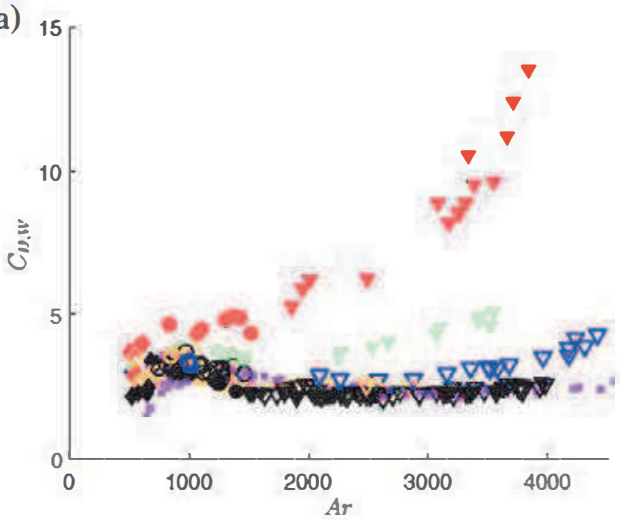

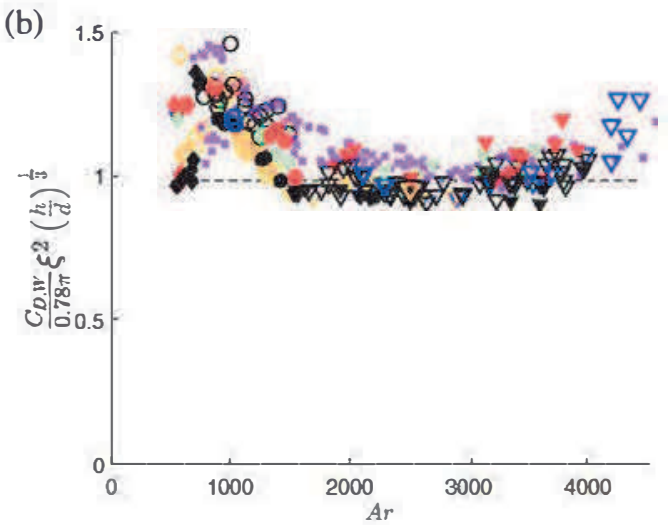

FIG. 14. (a) $C_{D, W}$ and (b) $C_{D, W}$ normalized with expression (A5), characterizing the drag experienced by a rising oscillating bubble for different cell widths $W(h \simeq 2.8 \mathrm{~mm})$, as functions of the Archimedes number of the bubble. Same convention as in Fig. 2(a).

where $\mathscr{S}_{W}$ is the representative surface of the bubble opposing the liquid flow, $\mathscr{V}=\pi d^{2} h / 4$ is the bubble volume (neglecting the thickness of the thin liquid films separating the bubble and the gap walls), and $C_{D, W}$ is the drag coefficient associated with the confinement $W$. Assuming $\mathscr{S}_{\mathbb{W}}=d h$, the increase in the drag coefficient $C_{D, W}$ experienced by a bubble of given volume (i.e., of given diameter $d$ ) with $W$ can be determined and is shown in Fig. 14(a) for oscillating bubbles in regimes $I$ to $I I I$ (symbols $\diamond, \circ$, and $\nabla$ ). To further obtain a generic expression of $C_{D, W}$, we note that expression (Al) also holds for a bubble of equal volume in the absence of in-plane confinement (no effect of $W$ ), so that (A l) can also be written as

$$
\frac{1}{2} \rho_{l} \mathscr{S}_{W} C_{D, W} V_{b}^{2}=\frac{1}{2} \rho_{l} \mathscr{S}_{\infty} C_{D, \infty} V_{b, \infty}^{2}
$$

where $\mathscr{S}_{\infty}, C_{D, \infty}$, and $V_{b, \infty}$ are the characteristic surface, the drag coefficient, and the mean rise velocity of a bubble experiencing the same buoyancy force (i.e., having the same diameter $d$ ) in the absence of transverse confinement (i.e., in a cell that is sufficiently wide). The increase in the drag coefficient associated with the presence of the lateral walls is, therefore, given by

$$
\left(\frac{C_{D, W}}{C_{n \times}}\right)=\left(\frac{\mathscr{S}_{\infty}}{\mathscr{S}_{W}}\right)\left(\frac{V_{b, \infty}}{V_{h}}\right):
$$

Combining this equation with expression (10), and assuming $\mathscr{S}_{w} \simeq \mathscr{S}_{\infty}$, provides an expression for the drag coefficient of bubbles displaying path oscillations, which accounts for the effects of both gap and transverse confinements in the ranges investigated here,

$$
C_{D, W} \simeq C_{D, \infty}\left(\frac{W}{W-d}\right)^{2} \simeq 0.78 \pi\left(\frac{d}{h}\right)^{1 / 3}\left(\frac{W}{W-d}\right)^{2}
$$

which also reads

$$
C_{D, W} \simeq 0.78 \pi \xi^{-2}\left(\frac{d}{h}\right)^{1 / 3} .
$$

The values of $C_{D, W}$ normalized with the right-hand side of expression (A5) are shown in Fig. 14(b) for different $\mathrm{Ar}$ and $W$. The prediction is particularly satisfactory in the range $1500<\mathrm{Ar}<4000$, corresponding to regime $I I I(\nabla)$. 


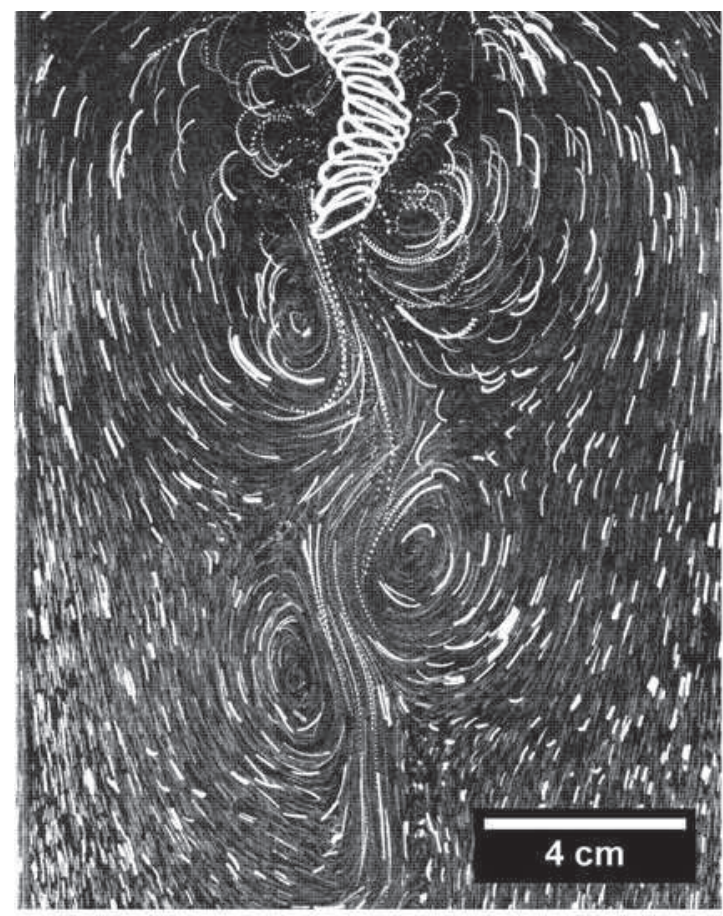

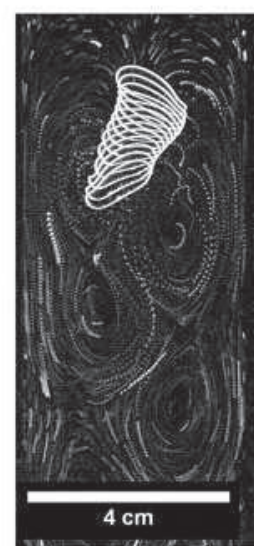

(b)

(a)

FIG. 15. Visualization of the vortex streets generated by a bubble with $\mathrm{Ar} \simeq 2650(h \simeq 2.8 \mathrm{~mm})$ in two cells having different widths [panel (a), $W=15 \mathrm{~cm}$; panel (b), $W=4.5 \mathrm{~cm}$ ], illustrating the difference in number of vortices released over the same distance. Both figures are plotted using the same scale, as indicated.

\section{APPENDIX B: EFFECT OF CONFINEMENT ON VORTEX SHEDDING}

To qualitatively verify the impact of confinement on vortex shedding for an oscillating bubble, we loaded the liquid phase with tracers and tracked their motion in time. This allowed us to visualize the vortex pattern associated with the bubble motion, for various $W$. An illustration of the result is provided in Fig. 15, for a bubble with $\mathrm{Ar} \approx 2650$ and two cell widths $W=4.5 \mathrm{~cm}$ and $W=15 \mathrm{~cm}$. The images were obtained by superposing several consecutive snapshots and were further processed in order to improve visibility. We can observe, in fact, that for the smallest cell width a larger number of smaller vortices accumulated over a given distance along the vertical direction.

[1] E. Alméras, F. Risso, V. Roig, C. Plais, and F. Augier, Mixing mechanism in a two-dimensional bubble column, Phys. Rev. Fluids 3, 074307 (2018).

[2] M. Roudet, A.-M. Billet, S. Cazin, F. Risso, and V. Roig, Experimental investigation of interfacial mass transfer mechanisms for a confined high Reynolds-number bubble rising in a thin gap, AIChE J. 63, 2394 (2017).

[3] C. Thobie, E. Gadoin, W. Blel, J. Pruvost, and C. Gentric, Global characterization of hydrodynamics and gas-liquid mass transfer in a thin-gap bubble column intended for microalgae cultivation, Chem. Eng. Process: Process Intensification 122, 76 (2017).

[4] F. Felis, F. Strassl, L. Laurini, N. Dietrich, A.-M. Billet, V. Roig, S. Herres-Pawlis, and K. Loubière, Using a bio-inspired copper complex to investigate reactive mass transfer around an oxygen bubble rising freely in a thin-gap cell, Chem. Eng. Sci. 207, 1256 (2019). 
[5] Z. Zhang, H. Zhang, X. Yuan, and K.-T. Yu, Effective UV-induced fluorescence method for investigating interphase mass transfer of single bubble rising in the Hele-Shaw cell, Ind. Eng. Chem. Res. 59, 6729 (2020).

[6] J. Pruvost, F. Le Borgne, A. Artu, and J. Legrand, Development of a thin-film solar photobioreactor with high biomass volumetric productivity (AlgoFilm(C) based on process intensification principles, Algal Res. 21, 120 (2017).

[7] F. Takemura, S. Takagi, J. Magnaudet, and Y. Matsumoto, Drag and lift forces on a bubble rising near a vertical wall in a viscous liquid, J. Fluid Mech. 461, 277 (2002).

[8] F. Takemura and J. Magnaudet, The transverse force on clean and contaminated bubbles rising near a vertical wall at moderate Reynolds number, J. Fluid Mech. 495, 235 (2003).

[9] H. Jeong and H. Park, Near-wall rising behaviour of a deformable bubble at high Reynolds number, J. Fluid Mech. 771, 564 (2015).

[10] B. Figueroa-Espinoza, R. Zenit, and D. Legendre, The effect of confinement on the motion of a single clean bubble, J. Fluid Mech. 616, 476 (2008).

[11] V. Roig, M. Roudet, F. Risso, and A.-M. Billet, Dynamics of a high-Reynolds-number bubble rising within a thin gap, J. Fluid Mech. 707, 444 (2012).

[12] A. Filella, P. Ern, and V. Roig, Oscillatory motion and wake of a bubble rising in a thin-gap cell, J. Fluid Mech. 778, 60 (2015).

[13] M. Hashida, K. Hayashi, and A. Tomiyama, Rise velocities of single bubbles in a narrow channel between parallel flat plates, Int. J. Multiphase Flow 111, 285 (2019).

[14] M. Hashida, K. Hayashi, and A. Tomiyama, Effects of fine particles on terminal velocities of single bubbles in a narrow channel between parallel flat plates, Int. J. Multiphase Flow 127, 103270 (2020).

[15] S. Piedra, E. Ramos, and J. R. Herrera, Dynamics of two-dimensional bubbles, Phys. Rev. E 91, 063013 (2015).

[16] X. Wang, B. Klaasen, J. Degrève, A. Mahulkar, G. Heynderickx, M.-F. Reyniers, B. Blanpain, and F. Verhaeghe, Volume-of-fluid simulations of bubble dynamics in a vertical Hele-Shaw cell, Phys. Fluids 28, 053304 (2016).

[17] X. Wang, B. Klaasen, J. Degrève, B. Blanpain, and F. Verhaeghe, Experimental and numerical study of buoyancy-driven single bubble dynamics in a vertical Hele-Shaw cell, Phys. Fluids 26, 123303 (2014).

[18] R. Collins, A simple model of the plane gas bubble in a finite liquid, J. Fluid Mech. 22, 763 (1965).

[19] M. Murano and K. Okumura, Rising bubble in a cell with a high aspect ratio cross-section filled with a viscous fluid and its connection to viscous fingering, Phys. Rev. Research 2, 013188 (2020).

[20] J. Bush and I. Eames, Fluid displacement by high Reynolds number bubble motion in a thin gap, Int. J. Multiphase Flow 24, 411 (1998).

[21] J. C. Cano-Lozano, C. Martínez-Bazán, J. Magnaudet, and J. Tchoufag, Paths and wakes of deformable nearly spheroidal rising bubbles close to the transition to path instability, Phys. Rev. Fluids 1, 053604 (2016).

[22] G. Birkhoff and D. Carter, Appendix D of LA-1927, Taylor instability, Appendices to Report LA-1862, Los Alamos, 1955.

[23] R. M. Davies and G. I. Taylor, The mechanics of large bubbles rising through extended liquids and through liquids in tubes, Proc. R. Soc. London Ser. A 200, 375 (1950).

[24] C. Clanet, P. Héraud, and G. Searby, On the motion of bubbles in vertical tubes of arbitrary cross-sections: some complements to the Dumitrescu-Taylor problem, J. Fluid Mech. 519, 359 (2004).

[25] A. Filella, Mouvement et sillage de bulles isolées ou en interaction confinées entre deux plaques, Ph.D. thesis, Institut de Mécanique des Fluides de Toulouse, Université de Toulouse, 2015. 